\title{
Visualizing and Interpreting Rhythmic Patterns Using Phase Space Plots
}

Andrea Ravignani

Vrije Universiteit Brussel, Brussels, Belgium

Max Planck Institute for Psycholinguistics, Nijmegen, The Netherlands

STRUCTURE IN MUSICAL RHYTHM CAN BE MEASURED using a number of analytical techniques. While some techniques-like circular statistics or grammar induction-rely on strong top-down assumptions, assumption-free techniques can only provide limited insights on higher-order rhythmic structure. I suggest that research in music perception and performance can benefit from systematically adopting phase space plots, a visualization technique originally developed in mathematical physics that overcomes the aforementioned limitations. By jointly plotting adjacent interonset intervals (IOI), the motivic rhythmic structure of musical phrases, if present, is visualized geometrically without making any a priori assumptions concerning isochrony, beat induction, or metrical hierarchies. I provide visual examples and describe how particular features of rhythmic patterns correspond to geometrical shapes in phase space plots. I argue that research on music perception and systematic musicology stands to benefit from this descriptive tool, particularly in comparative analyses of rhythm production. Phase space plots can be employed as an initial assumption-free diagnostic to find higher order structures (i.e., beyond distributional regularities) before proceeding to more specific, theory-driven analyses.

Received: June 17, 2016, accepted January 3, 2017.

Key words: rhythm, isochrony, timing, interonset intervals, nPVI

S EVERAL METHODS HAVE BEEN PROPOSED TO quantify structure and complexity in the production of rhythmic patterns. Most methods, however, are either unsuited to capture structural regularities or can only be employed after making top-down assumptions about the musical signal (Essens \& Povel, 1985; Longuet-Higgins \& Lee, 1982, 1984; Povel \& Essens, 1985; Shmulevich \& Povel, 2000). For instance, circular statistics are ideal to test for isochrony or isochronous synchronization (Clayton, 2007; Cook, Rouse, Wilson, \& Reichmuth, 2013; Large \& Gray, 2015; Moens et al., 2014; Rankin, Large, \& Fink, 2009; Ravignani \& Norton, 2017), but only when a stable, metronome-like reference signal can be assumed. Likewise, grammar induction techniques are excellent at capturing rhythmic structure, but they rely on strong top-down assumptions (Fisher, 1995; GranrothWilding \& Steedman, 2014). Here, I argue for the benefits of employing phase space plots as a descriptive tool in the analysis of rhythm production, and provide a tutorial on their use in musical rhythm research. In addition, I offer multiple visual examples and theoretical generalizations to show how various rhythmic patterns map to specific geometrical shapes in phase space plots.

Using phase space plots as diagnostic tools in rhythm experiments has many benefits, in particular aiding the selection of the most appropriate statistical techniques to perform the actual data analysis. Particular empirical cases where phase space plots might be useful are:

- Tapping tasks such as a synchronizationcontinuation task: the plot provides an instantaneous overview, for instance, of possible speeding up vs. slowing down behaviors;

- Tasks that involve sequential imitation of musical rhythm without an explicit isochronous pulse (provided by a metronome or a rhythmic instrument);

- Tasks involving simultaneous imitation of a musical rhythm: comparing the phase space plots of model and observer readily provides an assessment of imitation fidelity;

- Expressive performance studies: comparing the geometric regularity of a score-generated plot with possible deviations from it in the performancegenerated plot can provide a quick assessment of how mechanical a performance is;

- Analysis of improvisation complexity, such as in jazz music: improvisational devices such as retrogression, repeating riffs, and circular permutations can be visually recognized in phase space plots;

- Spontaneous production of musical patterns in infants or children with no music training: as no assumptions about musical enculturation can be

Music Perception, volume 34, issue 5, pp. 557-568, issn 0730-7829, electronic issn 1533-8312. C 2017 by the Regents of the university of California All RIGHTS RESERVED. PLEASE DIRECT ALL REQUESTS FOR PERMISSION TO PHOTOCOPY OR REPRODUCE ARTICLE CONTENT THROUGH THE UNIVERSITY OF CALIFORNIA PRESS'S Reprints and Permissions web page, http://www.ucpress.edu/journals.Php?P=Reprints. DOI: https://doi.org/10.1525/mp.2017.34.5.557 
made, phase space can provide an assumptionfree, initial screening of the data;

- All other kinds of rhythm perception and production experiments: phase space plots might unveil additional information beyond the theory-driven hypotheses originally intended to be tested.

\section{Distributional Measures of Temporal Structure}

Current methods used to analyze and visualize series of time intervals (McAuley, 2010), as produced in music, lack a unified framework. Series of time intervals are ubiquitous in music and movement research, deriving from spontaneous or experimentally driven rhythmic behavior in humans and other animal species. When confronted with such data, an initial approach so far has been to visualize the distribution of interonset intervals (IOIs) with a box plot (Figure 1, column i; Osman, Albert, Ridderinkhof, Band, \& van der Molen, 2006). This method may be fine for detecting simple features such as event isochrony (Figure 1, row A), but provides very limited information about regularities beyond isochrony if and when they are present. Another possible approach consists of plotting the histogram of the distribution of IOI (Figure 1, column ii; Allan \& Wiggins, 2006; Merchant, Zarco \& Prado, 2008). This method allows for visualizing how many of each type of intervals are present and can, thus, convey some information about the underlying rhythmic structure. For instance, a normal distribution suggests some degree of event isochrony (Large \& Gray, 2015; Zentner \& Eerola, 2010; Ravignani \& Norton, 2017), with a sharper peak and thinner tails implying a more metronomic-like acoustic pattern (Figure 1, row A, column ii). A multimodal distribution (Figure 1, rows B-F, column ii) suggests that notes are sampled from several durational categories, instead of a less musical sampling from a continuous distribution of durational values (Desain \& Honing, 2003; Ravignani, Delgado, \& Kirby, 2016; Savage, Brown, Sakai, \& Currie, 2015). A uniform distribution suggests a lack of durational categories or higher-order structure (Figure 1, row G). Unfortunately, distributions close to uniform may also emerge when complex structure is present but several durational categories for notes exist (Figure 1, row D already shows a quite uniform distribution with only three durational values). An additional issue is that histogram-based inference heavily depends on the bin size, i.e., resolution, of the histogram. ${ }^{1}$

\footnotetext{
${ }^{1}$ After a histogram has been plotted, it is possible to test hypotheses on whether the empirical IOI distribution significantly differs from one expected theoretically (Patel, Iversen, Bregman, \& Schulz, 2009a,
}

Figure 1 shows six rhythmic patterns, each visualized in four possible ways. All plots were generated from the music notation (lower part of column i) via a Python script that repeated the pattern 50 times, adding some random Gaussian noise to render the plots more realistic. The patterns shown are: (A) an isochronous sequence; (B) a short sequence of three durations; (C) a more complex five-note sequence, the "Clave Son" (Toussaint, 2002, 2013); (D) a sequence obtained by adding a sixth durational value to the Clave Son; (E) a five-note sequence obtained by combining the threenote pattern in B and the two-note pattern in Figure 3A; (F) a motif obtained by swapping the order of two adjacent notes from pattern E. Note that patterns E \& F have similar box plots and histograms but differ drastically in phase space plots; $(\mathrm{G})$ a completely random sequence of IOI, obtained by uniformly and independently sampling each IOI between 0.9 and 3.1. In general, any random permutation of the same set of IOIs composing a pattern will provide identical distributional graphs (columns i-ii) but different structural graphs (columns iii-iv). Notice how one-dimensional histograms (ii) can be obtained by simply projecting the points of a phase space plot (iv) onto one of the axes and measuring their frequencies.

\section{Higher-order Measures of Temporal Structure}

Distribution-based approaches exclusively focus on the information obtainable by the statistical distribution of the data. In particular, zeroth-order distributions only provide information on the frequency of occurrence of specific values, rather than higher-order structural information. An example of a zeroth-order distributional metric is the Kolmogorov-Smirnov $D$, which measures the distance between an empirical and a theoretical distribution. Distribution-based approaches, like histograms and Kolmogorov-Smirnov $D$, are critically limited in that they only examine zeroth-order distributional structure: ${ }^{2}$ any random shuffling to the order of

2009b) using, for instance, the Kolmogorov-Smirnov test (Allan \& Wiggins, 2006; Kolmogorov, 1933; Smirnov, 1948). This test is based on the Kolmogorov-Smirnov $D$ statistic, which is a number quantifying how different two distributions are. In other words, Kolmogorov-Smirnov $D$ can be used as a zeroth-order distributional measure for, among others, durational patterns.

${ }^{2}$ Strictly speaking, it is possible to construct higher-order distributions (i.e., conditional distributions). Thus, the real problem is that most classical approaches are "zeroth-order distributional" rather than distributional per se. A more fundamental problem with all sequential approaches to musical rhythm (regardless whether they are distributional or not, or if so, whether they are low or high order) is that they ignore hierarchical properties of musical meter and rhythm. 

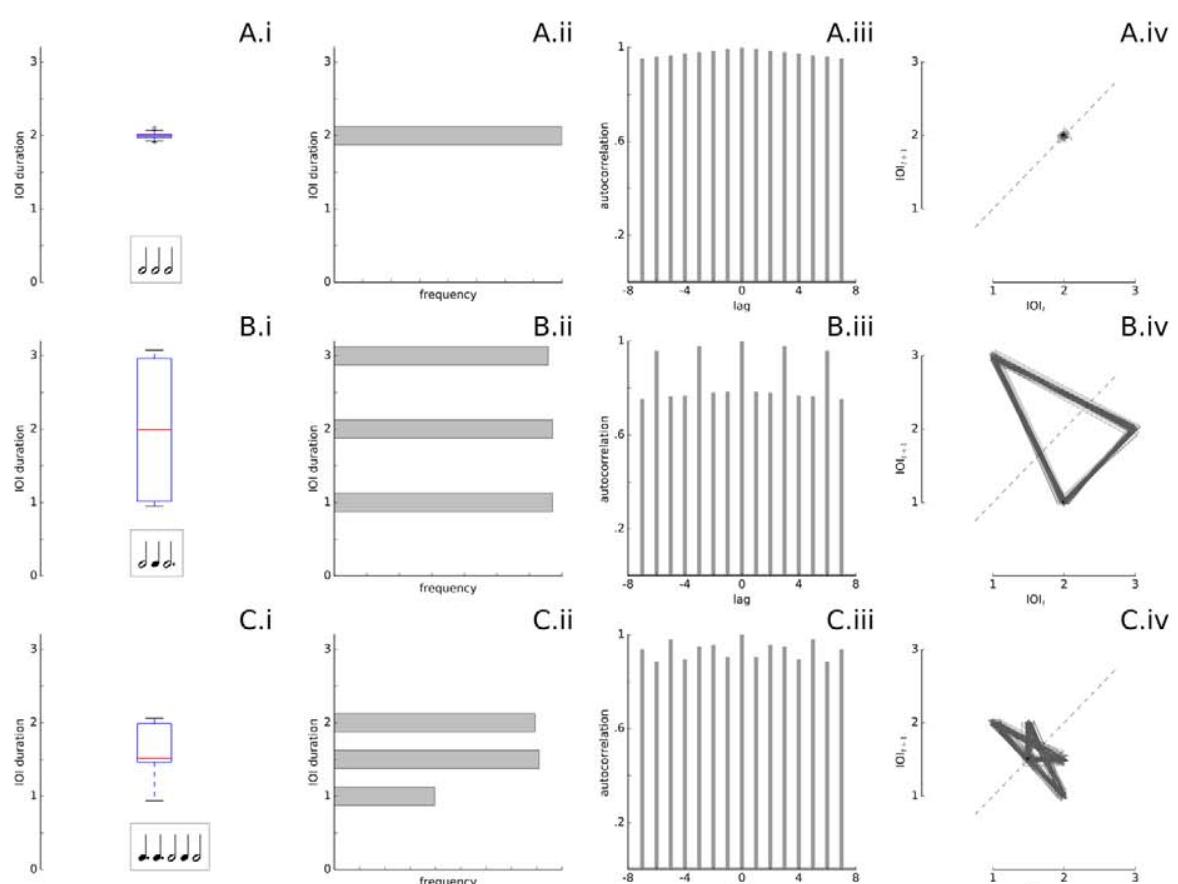

C.i
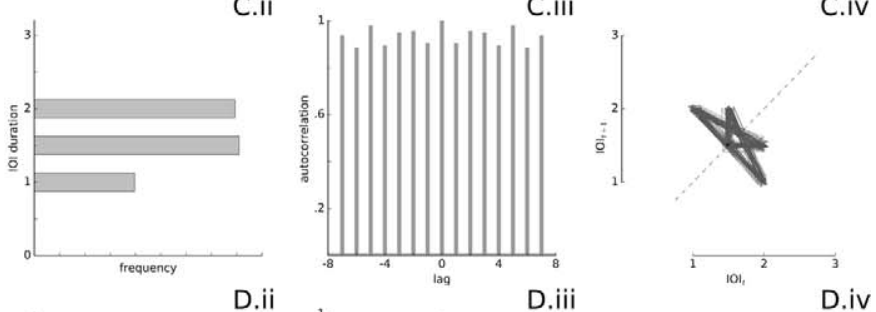

|
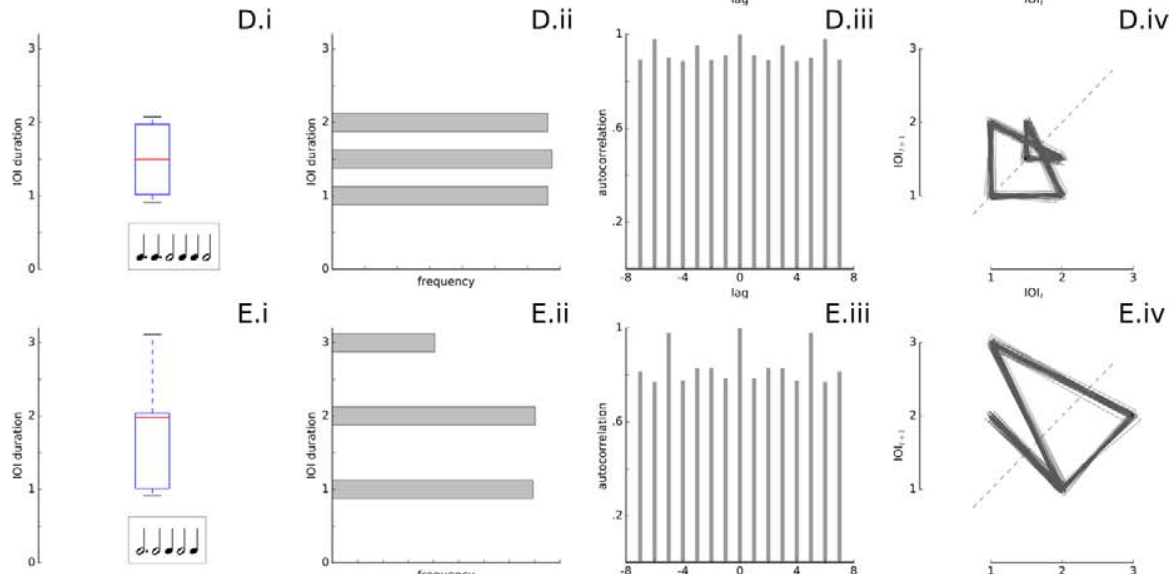

E.i
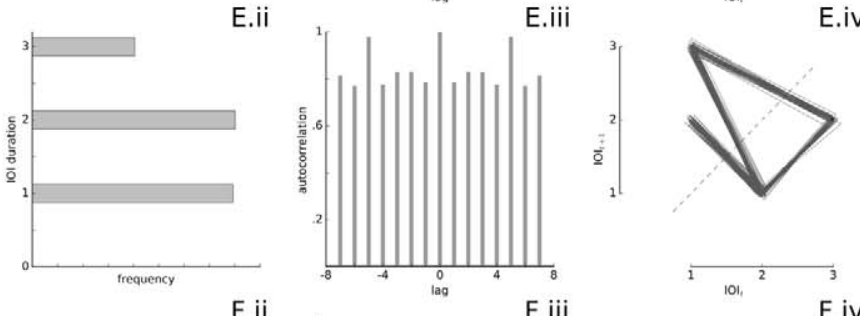

F.i

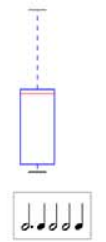

F.ii
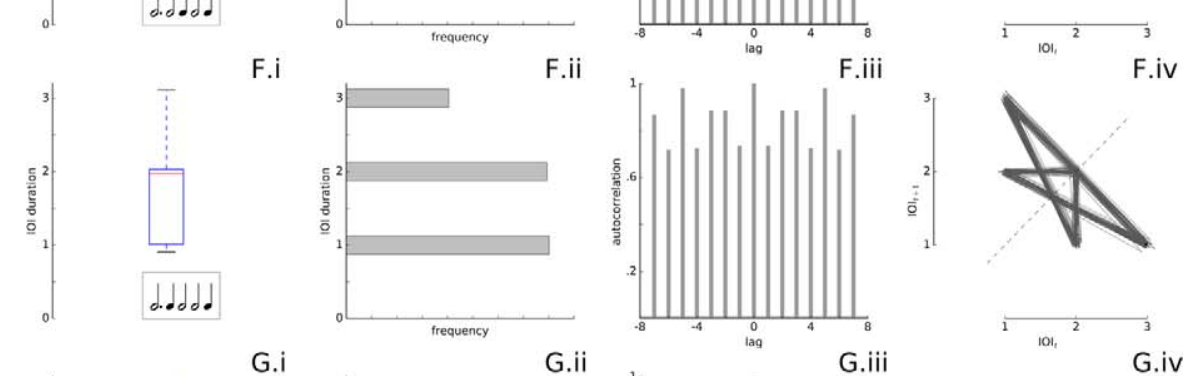

G.i
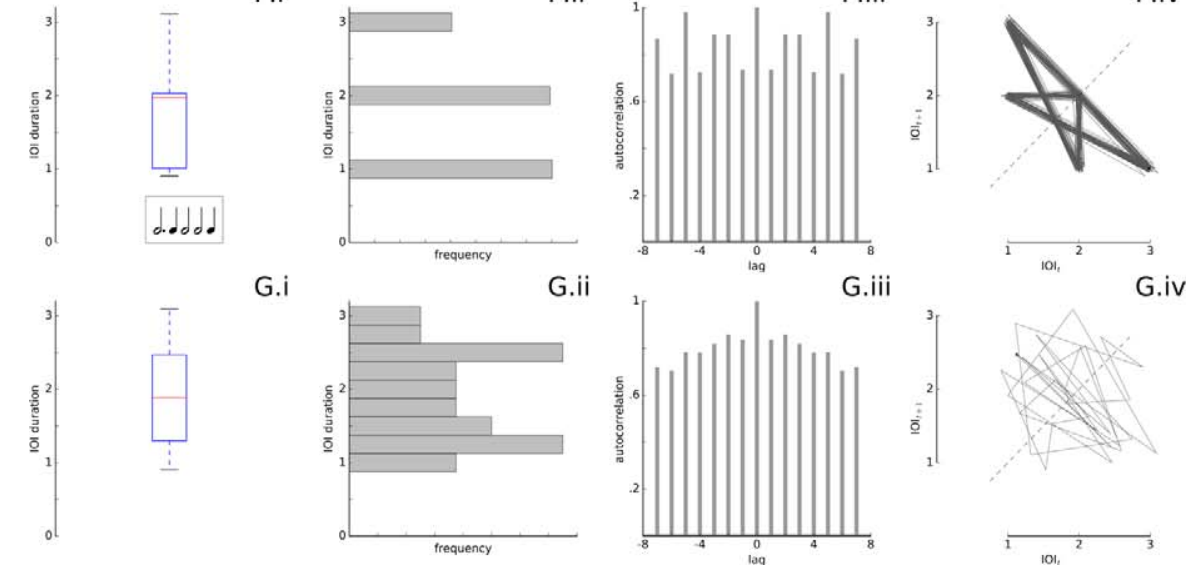

FIGURE 1. Six rhythmic patterns (rows A-F) depicted using boxplots (column i), histograms (column ii), autocorrelograms (column iii), and phase space plots (column iv). 
a series of IOIs (Figure 1, row E vs. F) can drastically change its rhythmic structure while leaving the box plot, Kolmogorov-Smirnov $D$ statistic, and histogram unaltered. In other words, box plots and histograms discard all information about regular co-occurrence of two or more IOIs. This information is not completely discarded when calculating the normalized pairwise variability index (nPVI), a common approach to the analysis of rhythm in music and speech (e.g., see Daniele \& Patel, 2015; Hansen, Sadakata \& Pearce, 2016; London \& Jones, 2011; Patel \& Daniele, 2003). This is a so-called firstorder method, as it goes beyond the mere distribution of durations composing a rhythmic pattern, calculating instead the degree of durational variability between adjacent IOIs. nPVI seems to explain more variance in the data than pure distributional metrics. Comparison of rhythm metrics on heterogeneous corpora shows some improvements of nPVI over the simple standard deviation (Toussaint, 2012), but not over other measures (Condit-Schultz, 2016).

Using a sample of 36 Western music excerpts composed between the 18th and 21st century and performed by string, woodwind, or brass instruments (von Coler \& Lerch, 2014), I calculated the nPVI and KolmogorovSmirnov $D$ for each piece. Kolmogorov-Smirnov $D$ (Kolmogorov, 1933; Smirnov, 1948) is a zeroth-order metric that simply measures the distance between two distributions: 1) an empirical distribution, in this case the IOI distribution for each music excerpt, and 2) a theoretical distribution, here a normal distribution with the same median and variance as the empirical. In other words, $D$ measures how normally distributed and close to isochrony the IOIs are. I found a significant correlation between $\mathrm{nPVI}$ and $D$ (Spearman rank-order correlation coefficient: $-.619, p<.001, N=36)$ suggesting that the broadly used (first-order) nPVI provides some extra explanatory power than the purely distributional (zeroth-order) measure $D .{ }^{3}$ Distributional methods only reflect which durational values occur and how often, while first-order methods discard all information beyond nearest neighbor relations. Fruitful attempts to complement pairwise variability with distributional information might lead to improved metrics (ConditSchultz, 2016). However, until a better replacement is found, nPVI seems the most empirically suitable approach to quantify rhythmic structure.

\footnotetext{
${ }^{3}$ Similar analyses for speech rhythm show that the purely distributional Kolmogorov-Smirnov $D$ strongly correlates with nPVI, which instead takes first-order (i.e., adjacent) structure into account (Jadoul, Ravignani, Thompson, Filippi, \& de Boer, 2016; Ravignani \& Norton, 2017).
}

Rhythmic patterns often exhibit higher structural complexity than the mere statistical distribution of IOIs (Toussaint, 2012). In time series, the existence of higher-order (i.e., non-local) patterns (Brockwell \& Davis, 2013; Hamilton, 1994) is typically examined using cross-correlations (in particular one of their visual counterparts, autocorrelograms: Figure 1, column iii) and autoregressive (AR) models (Ghazanfar, Takahashi, Mathur, \& Fitch, 2012; Jadoul, Ravignani, Thompson, Filippi, de Boer, 2016; Jones, 1980; Ravignani \& Norton, 2017; Wing, Endo, Bradbury, \& Vorberg, 2014). Unlike purely distributional approaches, these techniques can test for the existence of higher order structure (say the existence of adjacent and nonadjacent dependencies between durational categories) because they preserve information about the temporal order of IOIs (Clayton, 2007; Clayton, Sager, \& Will, 2005). Specifically, AR models test whether any given IOI is approximated by a linear combination (i.e., weighted sum) of previous IOIs. Autocorrelograms correlate a signal with itself at different delays, and show the magnitude of this autocorrelation for each lag value. A relatively high and constant autocorrelation across lags suggests isochrony (Figure 1A.iii). A few high and equally spaced values in the autocorrelation, separated by many lower values, suggest a lack of temporal periodicity in the signal (Figure 1, rows B-F, column iii). Autocorrelation is also particularly wellsuited to the discovery of "harmonic" relations among IOIs, (i.e., when durational values are related by integer multiples or divisors). However, systematically inferring a specific rhythmic structure (e.g., a repeating motif of several IOIs in a sequence) from autocorrelation plots alone is difficult. The primary reason is that complex rhythmic structures are not easily mapped to specific "autocorrelation landscapes" and vice versa (compare Figure 1, rows $C$ and $E$, with identical minima and maxima in autocorrelation but different rhythmic structure).

A rhythmic pattern of $n$ IOI can also be represented geometrically as a point in an $n$-dimensional space, where each interval duration corresponds to one dimension (Desain \& Honing, 2003; Honing, 2013). This representation is an elegant mathematical object, and has many advantages in the analysis of music perception and production. In particular, several geometrical features of this 'performance space' (Desain \& Honing, 2003) are isomorphic to the perceptual space of performed music. However, this method has two crucial limitations: (a) in 2D images it can only be used to visualize patterns composed of a maximum of 3 different IOIs, and (b) these IOIs must sum to the same predefined duration. It might be possible to extend the "performance space" to represent any fixed-length 
sequence of four IOIs using a three-dimensional object-namely a tetrahedron, or triangular pyramid-embedded in four dimensions. However, the limits of human visual perception put hard limits on the applicability of this and other higher dimensional approaches as intuitive diagnostic tools. (This visualization is not shown in Figure 1 because most example patterns could not be rendered in two dimensions.)

\section{Phase Space Plots: Capturing Higher-order Rhythmic Structure in Just Two Dimensions}

Each of the methods described above has its limitations. Moreover, no common method is broadly and universally employed to visualize rhythmic patterns in music perception and systematic musicology. Here I suggest that phase space plots, which are commonly used to detect higher order temporal patterns in dynamical systems theory, overcome some problematic issues in rhythmic pattern analysis and visualization, and can thus provide additional insights for researchers analyzing rhythm data (Figure 1, column iv). Dynamical systems theory is a powerful mathematical toolkit, originally developed in physics to characterize and quantify how systems of interacting entities evolve over time (Hunt, 2006; Pikovsky, Rosenblum, \& Kurths, 2003; Strogatz, 2003; Winfree, 1986, 2001). Mathematical tools from dynamical systems are now being successfully employed to analyze data in neuroscience, human movement science, and to some extent, systematic musicology (Konvalinka et al., 2011; Loehr, \& Palmer, 2007; Merker, Madison, \& Eckerdal, 2009; Ravignani \& Norton, 2017; Reiss \& Sandler, 2003). Phase space plots in particular have been successfully deployed to describe music in the melodic/harmonic domains (Gerhard, 1999; Monro \& Pressing, 1998; Rothenberg, Roeske, Voss, Naguib, \& Tchernichovski, 2014) and discover patterns for music information retrieval (Colucci, Chacón, \& Leguizamon, 2013; Wu \& Bello, 2010), but their usage in studies of rhythm perception and production has yet to reach its full potential. Moreover, the results of dynamical system analyses (Birkhoff, 1927; Pikovsky et al., 2003) are often presented in the empirical literature by avoiding presentation of model assumptions and mathematical details (Strogatz \& Stewart, 1993; Winfree, 1986), which is cumbersome for many readers (Boon \& Decroly, 1995; Konvalinka et al., 2011; Takens, 1981). However, some of these tools only require high school mathematical training and can be extremely useful. I argue that using phase space plots as descriptive and diagnostic tools in rhythm experiments (Merker et al., 2009; Ravignani \&
Norton, 2017) has many benefits, most prominently in aiding the selection of the most appropriate statistical techniques to perform the actual data analysis (e.g., using circular statistics if the phase space plots suggests isochrony, detrending the IOI series if the plots show an accelerando). This approach mirrors what is usually done with distributional methods in frequentist statistics, where box plots and histograms are used to explore the data sets before an appropriate statistical test can be selected. Below I provide a short tutorial on using phase space plots in musical rhythm research.

Given a two-dimensional Cartesian coordinate system and a rhythmic pattern, represented by a sequence of IOIs $\left(I O I_{1}, I O I_{2}, \ldots, I O I_{n}\right)$, one possible phase space plot representation can be drawn as follows. Pairs of adjacent IOI are used as $x-y$ coordinates and plotted on the Cartesian plane (Figure 2). Every point in the graph encodes the duration of one IOI, on the $x$-axis, and the following IOI, on the $y$-axis. Hence the first $(x, y)$ pair (Figure $2 \mathrm{~A})$ will be a point with coordinates $\left(I O I_{1}, I O I_{2}\right)$, the second pair (Figure $2 \mathrm{~B}$ ) will have coordinates $\left(I O I_{2}, I O I_{3}\right)$ and so on, until the final $(x, y)$ pair, plotted as a point with coordinates $\left(I O I_{n-1}, I O I_{n}\right)$. Contiguous $(x, y)$ coordinates can be joined by lines (Figure $2 \mathrm{~B}-\mathrm{D})$, producing polygons, or arrows depicting transitions, representing the flow of time. ${ }^{4}$ The 45 -degree line (dashed line in Figures 1, 2, 3, and 4) corresponds to the isochrony region of the space: single points on this "isochrony" line, or close enough to it, correspond to two adjacent IOI of equal length; clusters of points on the line correspond to an isochronous sub-pattern.

Practically, in music perception and performance research, two main sorts of musical signals might be turned into phase space plots. Data can be collected digitally (e.g., Desain \& Honing, 2003; Ravignani, Delgado, \& Kirby, 2016), using for instance a MIDI interface. Alternatively, data might be manually annotated from the signal, using an annotation software (e.g., Jadoul et al., 2016; von Coler \& Lerch, 2014). Both cases result in a list of absolute timestamps: subtracting pairwise adjacent timestamps provides the list of IOIs. Finally, once a list of IOIs is obtained, it is enough to convert it into an $\mathrm{R}$ vector, and input the function plot(vector[1: length(vector)-1], vector[2: length(vector)], type= "l") to obtain a basic phase space plot.

\footnotetext{
${ }^{4}$ Plotting phase space plots, also known as phase portraits, of time series is common in the physics of dynamical systems, where phase portraits are plotted either for adjacent or for non-adjacent intervals. Notice that, although the plots we present here where originally developed in Dynamical Systems Theory, analyzing timing in phase space does not, on its own, imply a Dynamical Systems Theory approach.
} 


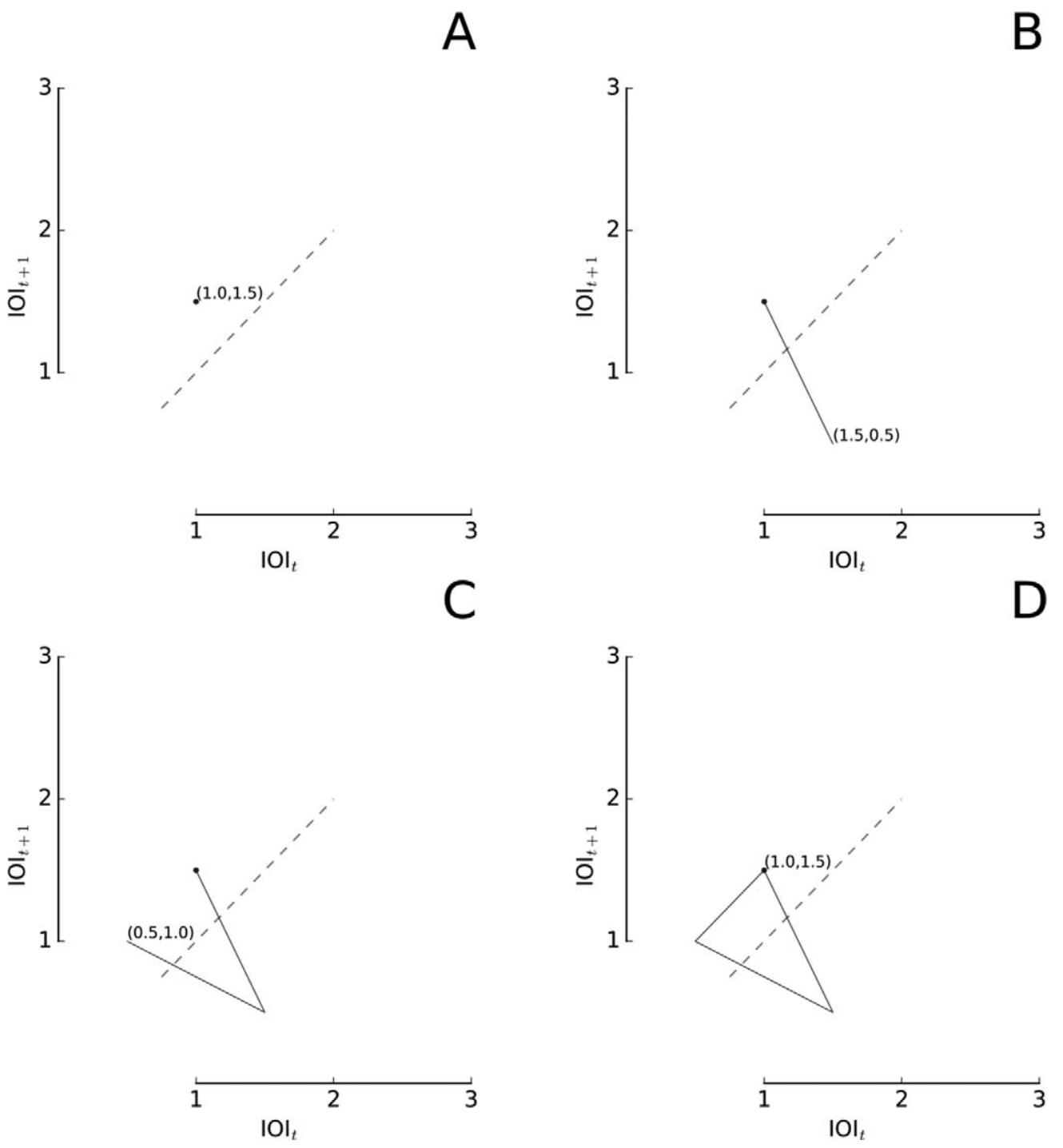

FIGURE 2. How to build and interpret a phase space plot step by step (A-D). The first IOI pair $(1,1.5)$ is depicted as a gray point $(A)$, showing the starting point of the line and hence of the rhythmic pattern. In (B), the previous y coordinate becomes the new $x$ coordinate, giving the $(1.5,0.5)$ pair. The procedure is iterated (C-D) until the end of the rhythmic pattern $(1,1.5,0.5,1)$. A more realistic and less mechanic performance, which includes some random noise, is depicted in Figure 3.

Once an IOI sequence is represented as a phase space plot, a number of inferences about the temporal rhythmic structure can be immediately drawn from its geometrical features (Table 1). First of all, a completely random pattern corresponds to uniformly distributed $(x, y)$ points (Figure 1G.iv); deviations from uniformity suggest the existence of some rhythmic structure. Two main types of geometrical shapes, possibly combined and nuanced, might be found: A cloud of points in close proximity corresponds to an isochronous subsequence (see Table 1 and Figure 1A.iv for details), and polygons correspond to specific motifs (e.g., the Clave son rhythm in Figure 1C.iv). In phase space coordinates, a repeated motif is a series of superimposed similar polygons (Figure 3, column ii). A musical piece composed by e.g., two sub-motifs is visually equivalent to the two corresponding polygons in Figure 1B.iv and 3A being connected (Figure 1E.iv). A series of similar polygons laid out along the isochrony 45 degree line (Figure 3 , column iv), corresponds to a repetition of the same motif while accelerando or ritardando (Table 1).

In addition to exploring the rhythmic structure in IOI sequences, phase space plots can also be used to draw inferences about structural similarities between two or 
TABLE 1. Correspondence Between Rhythmic Properties of Monophonic Temporal Patterns and Their Geometrical Representation in Phase Space Coordinates

\begin{tabular}{|c|c|c|}
\hline Rhythmic properties & Geometrical features & Figure example \\
\hline Isochrony & $\begin{array}{l}\text { Points' cloud clustered on the diagonal } \\
\text { (45-degree line) }\end{array}$ & 1A.iv \\
\hline Isochronous accelerando & Broken line on the diagonal towards origin & - \\
\hline Isochronous ritardando & Broken line on the diagonal towards + infinity & - \\
\hline $\begin{array}{l}\text { Number of non-identical } \\
\text { durational values }\end{array}$ & Number of vertices & $\begin{array}{r}1 \mathrm{~A} \text { (one duration), 3A (two), 1B, 3B (three), } \\
3 \mathrm{C} \text { (four), 1C.iv, 1E-F, 3D (five), 1D (six) }\end{array}$ \\
\hline $\begin{array}{l}\text { Pattern repeating } n \text { times almost } \\
\text { identically }\end{array}$ & $n$ almost identical polygons superimposed & 3A-D.ii \\
\hline Motif accelerando or ritardando & Similar polygons "smeared" along the diagonal & 3A-D.iii \\
\hline $\begin{array}{l}\text { Pulse rate (up to a multiplicative } \\
\text { constant) }\end{array}$ & Sum of all $\mathrm{x}$ coordinates of the polygon & - \\
\hline Symmetric pattern (e.g., 12321) & Symmetric with respect to the diagonal & $1 \mathrm{C}, 1 \mathrm{D}, 1 \mathrm{~F}, 3 \mathrm{~A}, 3 \mathrm{C}, 3 \mathrm{D}$ \\
\hline
\end{tabular}

more IOI sequences. Rhythmic similarities between two sequences may be often reflected in geometric similarities between their phase space plots (Table 2). This geometric comparison has advantages over box plots and/or other distributional visualizations. For example, different rhythms may appear similar when assessed using distributional measures (Figure 1E vs. 1F), but obviously differ when examined using phase space plots. Likewise, similar rhythmic patterns (Figure 1C vs. 1D) may produce different autocorrelograms due to the linearity of autocorrelation, but nevertheless similar polygons in phase space. Hence, while distributional measures discard important structural information, phase space plots provide, in the same amount of space, a mapping between rhythmic and geometric structures.

\section{Discussion}

Extraction of increasingly complex structural information from data often entails increasingly complex techniques. Distributional methods fail to capture higher-order structure, while circular statistics and grammar induction techniques rely on strong top-down assumptions (Fisher, 1995; Granroth-Wilding \& Steedman, 2014). This is not the case for phase space plots. Even if only visually, these plots can capture some global and local information in rhythmic patterns, clearly showing the presence of motifs. Moreover, their usage and geometrical interpretation is extremely simple, requiring little mathematics. For these reasons, I argue that phase space plots can offer systematic benefits at extremely low cost in experimental research on music production, entrainment, and human movement as an initial diagnostic tool. Beyond the benefits described above, this could systematize the analysis of rhythm production across experiments, which would be particularly useful in studies examining groups of two or more participants (Clayton, 2007), cross-species comparisons (which should be free from top-down assumptions; Ravignani, Fitch, et al., 2016; Wilson \& Cook, 2016), and crosscultural convergence of rhythmic features (Trehub, 2015) either in different musical cultures (Le Bomin, Lecointre, \& Heyer, 2016) or in musical experiments in the lab (Ravignani, Delgado, \& Kirby, 2016), all of which are core components of modern attempts to understand the biology and evolution of music (Fitch, 2006; Ravignani \& Cook, 2016).

\section{Limitations}

Visualization of rhythmic patterns using phase space plots of IOIs has some limitations. For instance, it focuses on durations, rather than absolute timing, so that, for instance, a pattern and its syncopated version will share an identical representation in phase space (Fitch \& Rosenfeld, 2007; see also Merker, 2014).

The scope of phase space plots is to visualize patterns as exploratory analysis (Pikovsky et al., 2003) in order to select an appropriate metric. There is a crucial difference between purely visual methods (like phase space plots) and timing metrics (like nPVI). Unlike phase space plots, metrics like nPVI and KolmogorovSmirnov $D$ provide numeric values that can been used to quantify differences in durational variability between music from different countries (Patel \& Daniele, 2003) and to track historical developments in compositional style (Hansen, Sadakata, \& Pearce, 2016). Graphical methods are not sufficient to address this kind of question, and should be used as a complement to quantitative methods. Although phase space plots are 
A.i

A.ii

A.iii
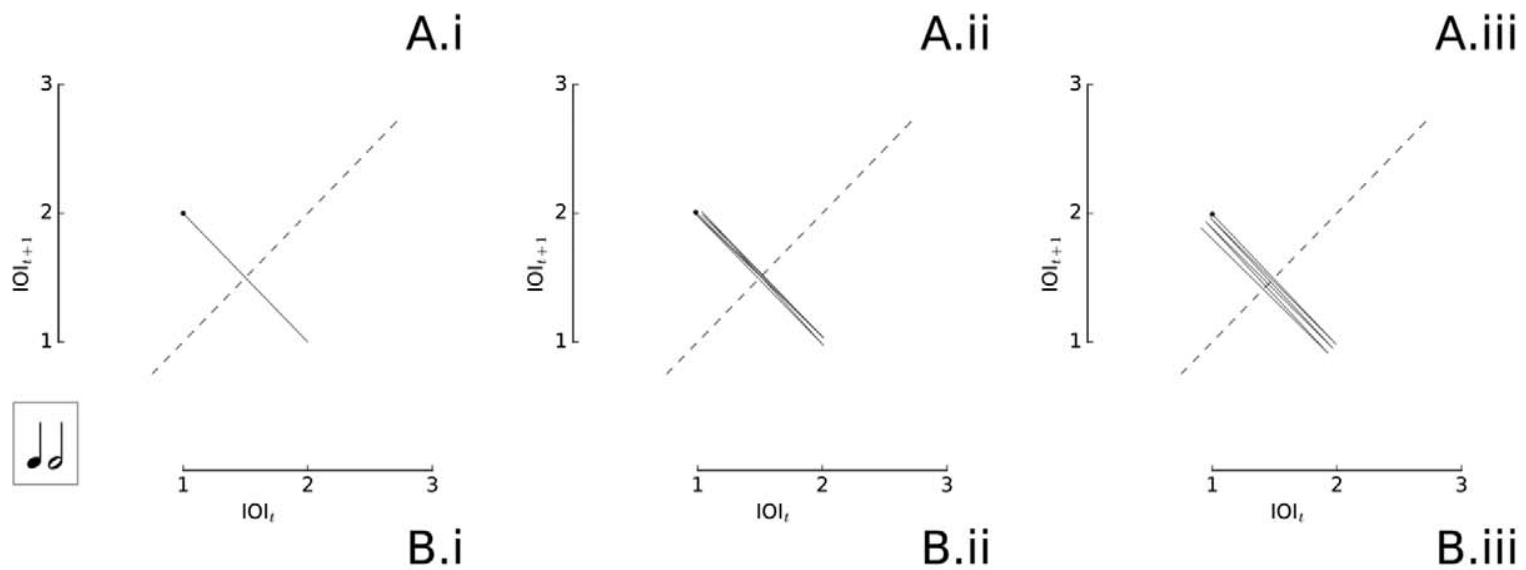

B.i

B.ii
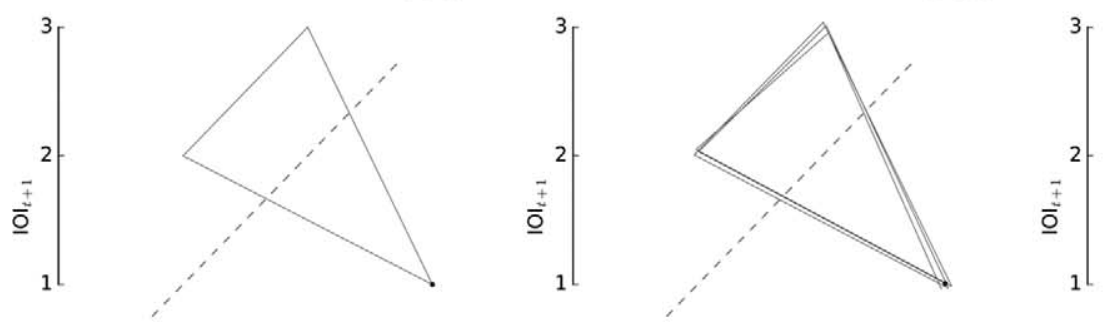

B.iii
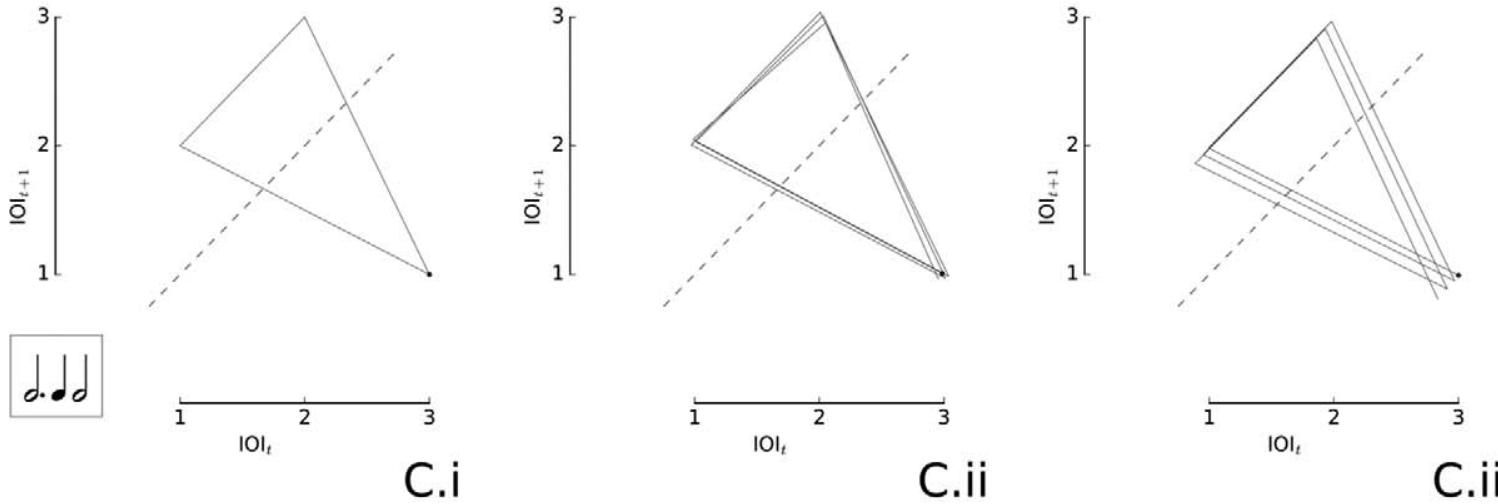

C.i
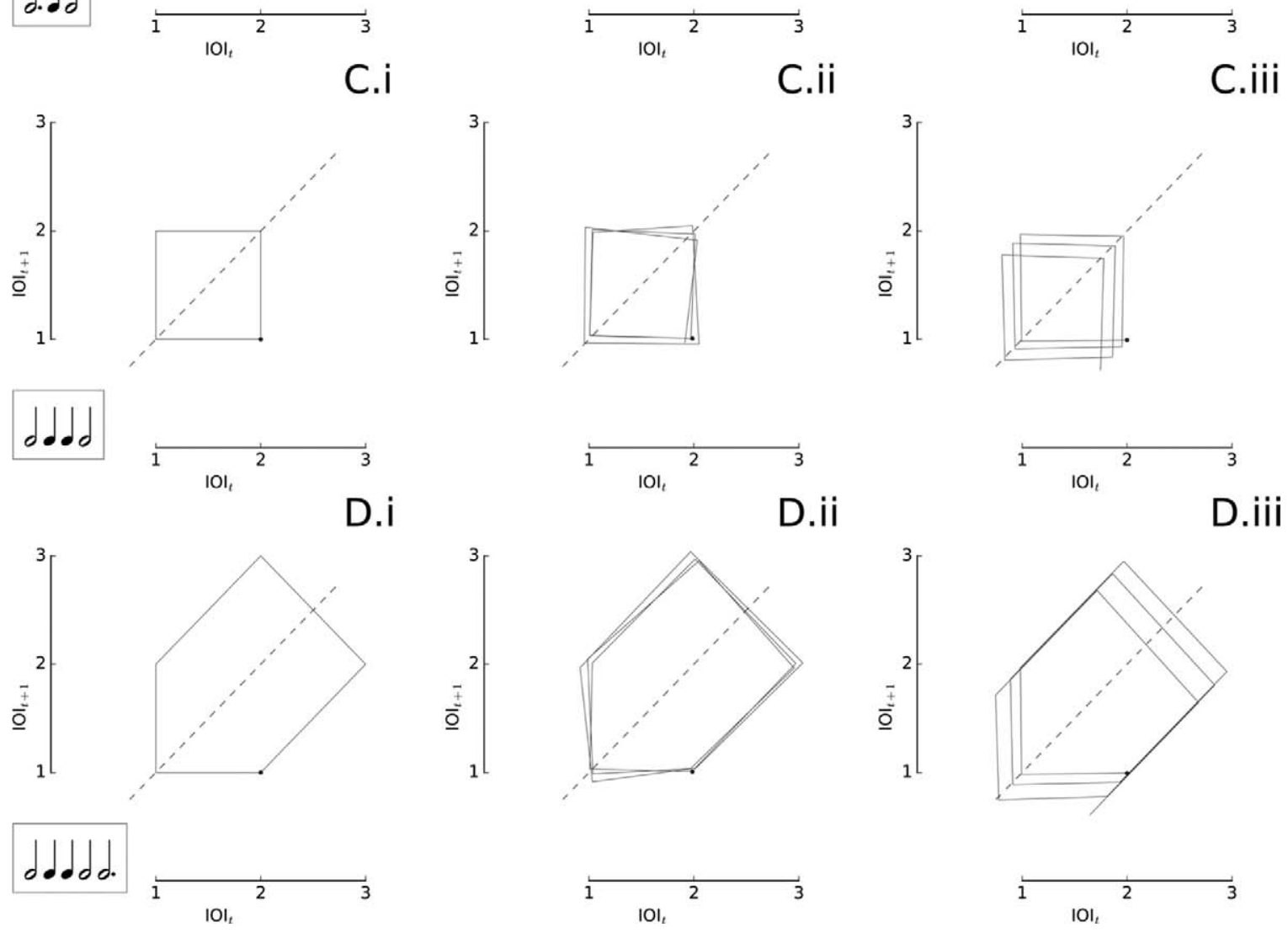

FIGURE 3. Phase space plots of durational structure in rhythmic patterns. One iteration (column i), and three iterations with random noise (similar overlaid polygons, column ii) and accelerando (similar smeared polygons, column iii) (Repp, London, \& Keller, 2012). The number of edges in a polygon describes the number of IOIs in the temporal sub-pattern, in this case: two notes of alternating length (row $A$ ); repeating patterns of length three (B), four (C), and five (D) IOIs. 
TABLE 2. Possible Relationships Between Two or More Monophonic Patterns

\begin{tabular}{|c|c|c|}
\hline Relational rhythmic properties & Pairwise geometrical features & Figure example \\
\hline $\begin{array}{l}\text { Synchronous patterns, or phase-shifted identical } \\
\text { patterns (possibly non-isochronous) }\end{array}$ & Same shapes, same location & - \\
\hline Same rhythmic sub-pattern of length $k+1$ & $\mathrm{k}$ consecutive vertices in common & $3 \mathrm{C}$ vs. $3 \mathrm{D}$ \\
\hline $\begin{array}{l}\text { One pattern is retrogression of the other } \\
\text { (e.g., } 123 \text { 321) }\end{array}$ & $\begin{array}{l}\text { One shape is the rotation of the other with respect to } \\
\text { the diagonal }\end{array}$ & 1B.iv vs. 3B \\
\hline Same patterns, different tempos & One shape is the translated and scaled version of the other & $2 \mathrm{D}$ vs. $3 \mathrm{~B}$ \\
\hline $\begin{array}{l}\text { Identical patterns, different initial IOI } \\
\quad \text { (e.g., } 12342341)\end{array}$ & Identical shapes & - \\
\hline Same set of IOI, different order & Different shapes & 1E.iv vs. 1F.iv \\
\hline
\end{tabular}

introduced here as a purely graphical method, future research should aim at quantifying similarities between plots, using advanced mathematical analysis (such as Lyapunov exponent) or geometrical techniques (such as Procrustes analysis).

As the plotted patterns grow in length, phase space plots might become harder to interpret. A possible solution to this may be to generate a new plot for every fixed-length subsequence of the whole pattern.

All compact summaries of empirical data involve a loss of information, and phase space plots are no exception to this limitation. A vast set of sequences, of which some are conspicuously different perceptually, do not receive visually distinct representations in the phase space. In other words, it is not the case that each distinct rhythmic pattern has a different plot shape. For example, almost all sequences composed of only quarter notes and half notes, irrespective of their ordering, will trace the square depicted in Figure 3ci, except when regular alternation between the two note values occurs. Similarly, a musical embellishment-like replacing a quarter note with two eighth notes-will modify two vertices of the geometrical shape in the plot. There is, in other words, no one-to-one correspondence between plot shapes and rhythmic patterning.

In human perception of musical rhythms, IOIsincluding the length of a note and a potential pauseare more cognitively relevant than the lengths of the note alone (e.g., Parncutt, 1994). Hence, while phase space plots focus on a cognitively relevant property, they are nonetheless blind to representing pauses.

\section{Future Work}

These and other possible limitations notwithstanding, there are several ways in which phase-space plots could be extended to increase their usefulness in rhythm analysis. Thus, they could easily be extended by introducing
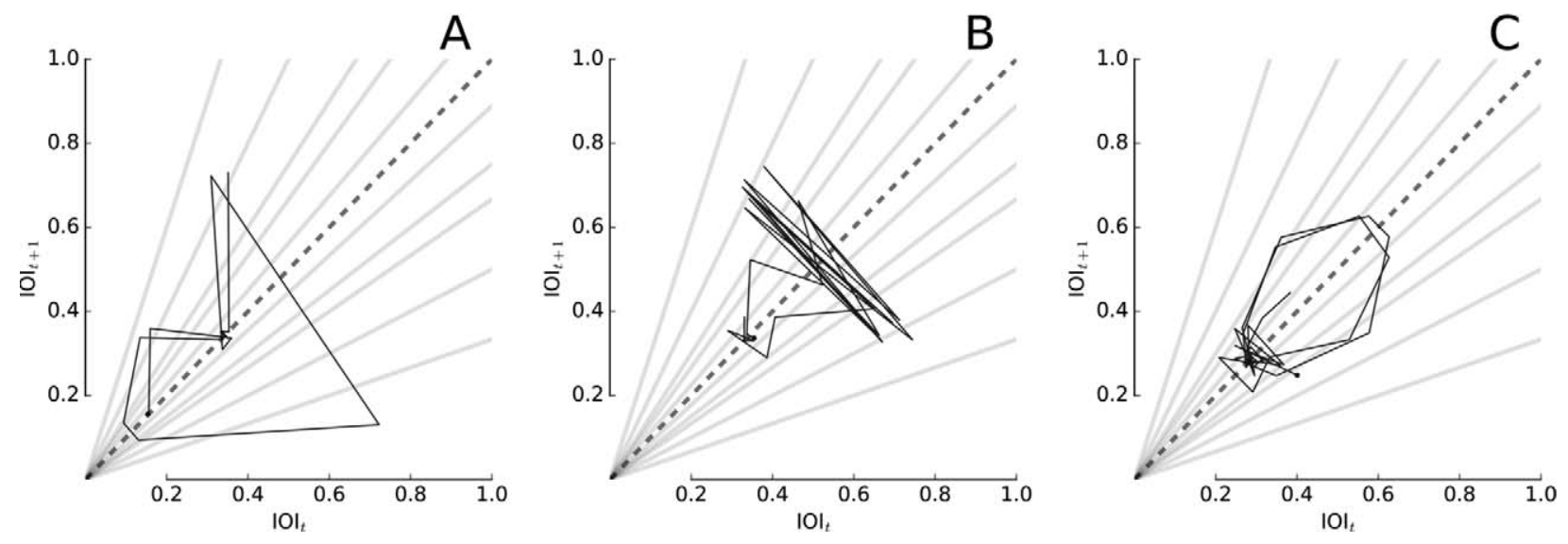

FIGURE 4. Phase space plots of short musical excerpts from three Western art musical pieces (von Coler \& Lerch, 2014): (A) S. Wolpe's Solo Piece for Trumpet: I.; (B) Fanfare for Solo Trumpet, composed by S. Beamish; (C) J.S. Bach's Cello Suite Nr.1-5, Menuet II. The integer ratio grids represent, clockwise 3:1, 2:1, 3:2, 4:3, 9:8, 1:1, and their reciprocals. Notice how: (A) lacks a clear polygonal shape but its IOI values are mostly in 2:1 (second line clockwise) and 1:1 (broken line) ratios; $(B)$ shows a short-long repeating pattern indicated by the cluster of diagonal lines, similar to Figure $3 A$; $(C)$ includes two overlaid hexagons, corresponding to a rhythmic motif of length 6 repeating twice. 
spectral measures to provide information about note accentuation and meter (London, 2012). For example, the points denoting two adjacent IOIs in the phase space plots could be color coded, so that darker shades of grey denote higher intensity differences between adjacent IOIs. This would provide the opportunity to visualize not only durational, but also accentual structures in phase space coordinates.

Moreover, phase space plots could be enriched by adding: 1) a grid of integer ratios, e.g., 1:4, 1:3 1:2, 2:3 (Merker, 2014) to visualize whether participant are producing metric structures and groupings (Motz, Erickson, \& Hetrick, 2013; Repp et al., 2012; Repp, London, \& Keller, 2013), and 2) "isoperceptual curves," that is, all partitions of the space for which the ratio between adjacent IOIs is constant, enabling inclusion of insights from Weber's law and scalar timing theory (Grondin, 2010; Matthews \& Meck, 2016; Weber, 1834). Figure 4 shows a first attempt at an integer ratio grid-taking Weber's constancy of ratios into account-overlaid on phase space plots of three musical excerpts.

So-called "Poincare sections" at different delays could be plotted to uncover hierarchical rhythmic structure. In other words, plotting $I O I_{t}$ vs. $I O I_{t+p}$ for a range of $p$ could show structural regularities at two levels: subpatterns of length $\mathrm{p}$ and their structural combination into a longer pattern.

Finally, phase space plots could be used as a simple compositional tool. In fact, reversing the mapping between IOI sequences and phase space plots, simple software could be developed to transform geometrical figures drawn on a touchscreen instantly into repeating rhythmic patterns. Overall, phase space plots promise to enhance comparability of studies in music perception and production across species, domains, and subdisciplines of research.

\section{Author Note}

Andrea Ravignani was supported by European Research Council grant 283435 ABACUS to Bart de Boer. The funders had no role in study design, decision to publish, or preparation of the manuscript. The author declares no competing financial interests.

Andrea Ravignani is grateful to Bart de Boer, Daniel Bowling, Tania Delgado, Piera Filippi, Tecumseh Fitch, Thomas Grossi, Yannick Jadoul, Simon Kirby, Alexandre Celma Miralles, Philipp Norton, Vittoria Spinosa, Yi-Huang Su, Bill Thompson, and Peeter Tinits for fruitful comments and discussions. The author sincerely thanks Lola Cuddy, Niels Christian Hansen, Adriano Lameira, Peter Pfordresher, and one anonymous reviewer for precious insights during the review process.

Correspondence concerning this article should be addressed to Andrea Ravignani, Artificial Intelligence Lab, Vrije Universiteit Brussel, Pleinlaan 2, 1050 Brussels, Belgium. E-mail: andrea.ravignani@gmail.com

\section{References}

Allan, H., \& Wiggins, G. (2006). Further aspects of similarity. In Proceedings of the 2nd Digital Music Research Network Summer Conference (pp. 1-4). Leeds, UK: Leeds Metropolitan University.

Birkhoff, G. D. (1927). On the periodic motions of dynamical systems. Acta Mathematica, 50, 359-379.

Boon, J. P., \& Decroly, O. (1995). Dynamical systems theory for music dynamics. Chaos: An Interdisciplinary Journal of Nonlinear Science, 5(3), 501-508.

Brockwell, P. J., \& Davis, R. A. (2013). Time series: Theory and methods. New York: Springer-Verlag.

Clayton, M. (2007). Observing entrainment in music performance: Video-based observational analysis of Indian musicians' tanpura playing and beat marking. Musicae Scientiae, 11, 27-59.

Clayton, M., Sager, R., \& Will, U. (2005). In time with the music: The concept of entrainment and its significance for ethnomusicology. European Meetings in Ethnomusicology, 11, 1-82.

Colucci, R., Chacón, G. R., \& Leguizamon, C. (2013). Some ideas on nonlinear musical analysis. Applied Mathematical Sciences, 7(25-28), 1283-1301.
Condit-Schultz, N. (2016). Deconstructing nPVI. In Proceedings of the 14th International Conference on Music Perception and Cognition (pp. 800-804). San Francisco, CA: ICMPC.

Cook, P., Rouse, A., Wilson, M., \& Reichmuth, C. J. (2013). A California sea lion (Zalophus californianus) can keep the beat: Motor entrainment to rhythmic auditory stimuli in a non vocal mimic. Journal of Comparative Psychology, 127(2), 1-16.

Daniele, J. R., \& Patel, A. D. (2015). Stability and change in rhythmic patterning across a composer's lifetime. Music Perception, 33, 255-265.

Desain, P., \& Honing, H. (2003). The formation of rhythmic categories and metric priming. Perception, 32, 341-365.

Essens, P. J., \& Povel, D.-J. (1985). Metrical and nonmetrical representations of temporal patterns. Perception and Psychophysics, 37, 1-7.

FISHER, N. I. (1995). Statistical analysis of circular data. Cambridge, UK: Cambridge University Press. 
Fitch, W. T. (2006). The biology and evolution of music: A comparative perspective. Cognition, 100, 173-215.

Fitch, W. T., \& Rosenfeld, A. J. (2007). Perception and production of syncopated rhythms. Music Perception, 25, 43-58.

Gerhard, D. (1999). Audio visualization in phase space. Paper presented at the Bridges: Mathematical Connections in Art, Music and Science (pp. 137-144), Winfield, KS.

Ghazanfar, A. A., Takahashi, D. Y., Mathur, N., \& Fitch, W. T. (2012). Cineradiography of monkey lip-smacking reveals putative precursors of speech dynamics. Current Biology, 22, 1176-1182.

Granroth-Wilding, M., \& Steedman, M. (2014). A robust parser-interpreter for jazz chord sequences. Journal of New Music Research, 43, 355-374.

Grondin, S. (2010). Timing and time perception: A review of recent behavioral and neuroscience findings and theoretical directions. Attention, Perception, and Psychophysics, 72, 561-582.

Hamilton, J. D. (1994). Time series analysis. Princeton, NJ: Princeton University Press.

Hansen, N. C., Sadakata, M., \& Pearce, M. (2016). Nonlinear changes in the rhythm of European art music. Music Perception, 33, 414-431.

Honing, H. (2013). The structure and interpretation of rhythm in music. In D. Deutsch (Ed.), Psychology of music (3rd ed., pp. 369-404). London, UK: Academic Press.

Hunt, E. (2006). The mathematics of behavior. New York: Cambridge University Press.

Jadoul, Y., Ravignani, A., Thompson, B., Filippi, P., \& De Boer, B. (2016). Seeking temporal predictability in speech: Comparing statistical approaches on 18 world languages. Frontiers in Human Neuroscience, 10(586), 1-15.

Jones, R. H. (1980). Maximum likelihood fitting of ARMA models to time series with missing observations. Technometrics, 22, 389-395.

Kolmogorov, A. (1933). Sulla determinazione empirica delle leggi di probabilità. Giornale dell'Istituto Italiano degli Attuari, 4, 1-11.

Konvalinka, I., Xygalatas, D., Bulbulia, J., Schjødt, U., Jegindø, E.-M., Wallot, S., Et Al. (2011). Synchronized arousal between performers and related spectators in a firewalking ritual. Proceedings of the National Academy of Sciences, 108, 8514-8519.

Large, E. W., \& Gray, P. M. (2015). Spontaneous tempo and rhythmic entrainment in a bonobo (Pan Paniscus). Journal of Comparative Psychology, 129, 317-328.

Le Bomin, S., Lecointre, G., \& Heyer, E. (2016). The evolution of musical diversity: The key role of vertical transmission. PloS One, 11(3), e0151570, 1-17.

Loehr, J. D., \& Palmer, C. (2007). Cognitive and biomechanical influences in pianists' finger tapping. Experimental Brain Research, 178, 518-528.
London, J. (2012). Three things linguists need to know about rhythm and time in music. Empirical Musicology Review, 7(1-2), 5-11.

London, J., \& Jones, K. (2011). Rhythmic refinements to the nPVI measure: A reanalysis of Patel \& Daniele (2003a). Music Perception, 29, 115-120.

Longuet-Higgins, H. C., \& Lee, C. S. (1982). The perception of musical rhythms. Perception, 11, 115-128.

Longuet-Higgins, H. C., \& Lee, C. S. (1984). The rhythmic interpretation of monophonic music. Music Perception, 1, 424-441.

Matthews, W. J., \& Mecк, W. H. (2016). Temporal cognition: Connecting subjective time to perception, attention, and memory. Psychological Bulletin, 142, 865-907.

McAuley, J. D. (2010). Tempo and rhythm. In M. Riess Jones, R. R. Fay, \& A. N. Popper (Eds.), Music Perception (pp. 165-199). New York: Springer New York.

Merchant, H., Zarco, W., \& Prado, L. (2008). Do we have a common mechanism for measuring time in the hundreds of millisecond range? Evidence from multiple-interval timing tasks. Journal of Neurophysiology, 99, 939-949.

Merker, B. (2014). Groove or swing as distributed rhythmic consonance: Introducing the groove matrix. Frontiers in Human Neuroscience, 8(454), 1-4.

Merker, B., Madison, G., \& Eckerdal, P. (2009). On the role and origin of isochrony in human rhythmic entrainment.

Cortex, 45, 4-17.

Moens, B., Muller, C., Van Noorden, L., FraněK, M., Celie, B., Boone, J., Bourgois, J., \& Leman, M. (2014).

Encouraging spontaneous synchronisation with D-Jogger, an adaptive music player that aligns movement and music. PloS One, 9(12), e114234.

Monro, G., \& Pressing, J. (1998). Sound visualization using embedding: The art and science of auditory autocorrelation. Computer Music Journal, 22(2), 20-34.

Motz, B. A., Erickson, M. A., \& Hetrick, W. P. (2013). To the beat of your own drum: Cortical regularization of non-integer ratio rhythms toward metrical patterns. Brain and Cognition, 81, 329-336.

Osman, A., Albert, R., Ridderinkhof, K. R., Band, G., \& Van Der Molen, M. (2006). The beat goes on: Rhythmic modulation of cortical potentials by imagined tapping. Journal of Experimental Psychology: Human Perception and Performance, 32, 986-1005.

Parncutt, R. (1994). A perceptual model of pulse salience and metrical accent in musical rhythms. Music Perception, 11, 409-464.

Patel, A. D., \& Daniele, J. R. (2003). An empirical comparison of rhythm in language and music. Cognition, 87, B35-B45.

Patel, A. D., Iversen, J. R., Bregman, M. R., \& Schulz, I. (2009a). Experimental evidence for synchronization to a musical beat in a nonhuman animal. Current Biology, 19, 827-830. 
Patel, A. D., Iversen, J. R., Bregman, M. R., \& Schulz, I. (2009b). Studying synchronization to a musical beat in nonhuman animals. Annals of the New York Academy of Sciences, 1169, 459-469.

Pikovsky, A., Rosenblum, M., \& Kurths, J. (2003). Synchronization: A universal concept in nonlinear sciences. New York: Cambridge University Press.

Povel, D.-J., \& Essens, P. (1985). Perception of temporal patterns. Music Perception, 2, 411-440.

Rankin, S. K., Large, E. W., \& Fink, P. W. (2009). Fractal tempo fluctuation and pulse prediction. Music Perception, 26, 401-413.

Ravignani, A., \& Cook, P. (2016). The evolutionary biology of dance without frills. Current Biology, 26, R878-R879.

Ravignani, A., Delgado, T., \& Kirby, S. (2016). Musical evolution in the lab: Rhythmic universals emerge in a cultural transmission experiment. Nature Human Behaviour, 1(0007), 1-7.

Ravignani, A., Fitch, W. T., Hanke, F. D., Heinrich, T., Hurgitsch, B., Kotz, Et Al. (2016). What pinnipeds have to say about human speech, music, and the evolution of rhythm. Frontiers in Neuroscience, 10(274), 1-9.

Ravignani, A., \& Norton, P. (2017). Measuring rhythmic complexity: A primer to quantify and compare temporal structure in speech, movement, and animal vocalizations. Journal of Language Evolution. DOI: 10.1093/jole/lzx002

Reiss, J., \& SANDLER, M. (2003). Nonlinear time series analysis of musical signals. Proceedings of the 6th International Conference on Digital Audio Effects DAFx-03 (pp. 1-5). London, UK: ICDAE.

Repp, B. H., London, J., \& Keller, P. E. (2012). Distortions in reproduction of two-interval rhythms: When the "attractor ratio" is not exactly 1: 2. Music Perception, 30, 205-223.

Repp, B. H., London, J., \& Keller, P. E. (2013). Systematic distortions in musicians: Reproduction of cyclic three-interval rhythms. Music Perception, 30, 291-305.

Rothenberg, D., Roeske, T. C., Voss, H. U., Naguib, M., \& Tchernichovski, O. (2014). Investigation of musicality in birdsong. Hearing Research, 308, 71-83.

Savage, P. E., Brown, S., Sakai, E., \& Currie, T. E. (2015). Statistical universals reveal the structures and functions of human music. Proceedings of the National Academy of Sciences, 112, 8987-8992.

Shmulevich, I., \& Povel, D.-J. (2000). Measures of temporal pattern complexity. Journal of New Music Research, 29, 61-69.

Smirnov, N. (1948). Table for estimating the goodness of fit of empirical distributions. The annals of mathematical statistics, 19, 279-281.
Strogatz, S. (2003). Sync: The emerging science of spontaneous order. New York: Hyperion.

Strogatz, S., \& Stewart, I. (1993). Coupled oscillators and biological synchronization. Scientific American, 269(6), 102-109.

TAKENS, F. (1981). Detecting strange attractors in turbulence. Dynamical Systems and Turbulence, Warwick 1980, Lecture Notes in Mathematics, 898, 366. Berlin: Springer-Verlag.

Toussaint, G. T. (2002). A mathematical analysis of African, Brazilian, and Cuban clave rhythms. Paper presented at the Proceedings of BRIDGES: Mathematical Connections in Art, Music and Science (pp. 157-168), Towson, MD.

Toussaint, G. T. (2012). The pairwise variability index as a tool in musical rhythm analysis. In Proceedings of the 12th International Conference on Music Perception and Cognition (ICMPC), and 8th Triennial Conference of the European Society for the Cognitive Sciences of Music (ESCOM) (pp. 1001-1008). Thessaloniki, Greece: ICMPC.

Toussaint, G. T. (2013). The geometry of musical rhythm: What makes a "good" rhythm good? Boca Raton, FL: CRC Press.

Trenub, S. E. (2015). Cross-cultural convergence of musical features. Proceedings of the National Academy of Sciences, 112, 8809-8810.

Von Coler, H., \& Lerch, A. (2014). CMMSD: A data set for note-level segmentation of monophonic music. Paper presented at the Audio Engineering Society Conference: 53rd International Conference: Semantic Audio (pp. 1-10). London, UK: AESC

Weber, E. H. (1834). De Pulsu, resorptione, auditu et tactu: Annotationes anatomicae et physiologicae. Leipzig, Germany: CF Koehler.

Wilson, M., \& Cook, P. F. (2016). Rhythmic entrainment: Why humans want to, fireflies can't help it, pet birds try, and sea lions have to be bribed. Psychonomic Bulletin and Review, 23, 1-13.

Winfree, A. T. (1986). Timing of biological clocks. New York: Henry Holt and Company.

Winfree, A. T. (2001). The geometry of biological time (Vol. 12). New York: Springer Science \& Business Media.

Wing, A. M., Endo, S., Bradbury, A., \& Vorberg, D. (2014). Optimal feedback correction in string quartet synchronization. Journal of The Royal Society Interface, 11(93), 20131125, 1-11.

Wu, H.-H., \& Bello, J. P. (2010). Audio-based music visualization for music structure analysis. Proceedings of Sound and Music Computing Conference (SMC) (p. 1-6). Barcelona, Spain: SMC.

Zentner, M., \& Eerola, T. (2010). Rhythmic engagement with music in infancy. Proceedings of the National Academy of Sciences, 107, 5768-5773. 